\title{
Pulmonale Hypertonie bei der Systemsklerose: Mono- vs. Kombinationstherapie
}

Pestaña-Fernández M et al. Longterm Efficacy and Safety of Monotherapy versus Combination Therapy in Systemic Sclerosis-associated

Pulmonary Arterial Hypertension: A Retrospective RESCLE Registry Study. J Rheumatol 2020; 47: 89-98. doi: 10.3899/jrheum.180595

Ein der gravierendsten Komplikationen der Systemsklerose ist die pulmonalarterielle Hypertonie, die unbehandelt zum Rechtsherzversagen und zum Tod führt. Therapeutisch stehen Endothelin-Rezeptor-Antagonisten (ERA) sowie Phosphodiesterase-5 (PDE5)-Hemmer zur Verfügung. Hat die Kombinationstherapie gegenüber der Monotherapie im Hinblick auf die Überlebensprognose Vorteile?

Dieser Frage gingen spanische Wissenschaftler im Rahmen einer retrospektiven Kohortenstudie nach. Sie werteten die Daten von 1817 Patienten mit einer Systemsklerose aus, die seit dem Jahr 2006 in das spanische Skleroderma-Register aufgenommen worden waren. Bei 76 Patienten (4,2\%) war mittels Rechtsherzkatheter eine pulmonalarterielle Hypertonie diagnostiziert worden. Gemäß der medikamentösen Therapiestrategie unterschieden die Forscher 3 Gruppen:

- Monotherapie mit ERA oder PDA5Hemmern

- Sequenzielle Kombinationstherapie (zwischen dem Start beider Medikamente vergingen $\geq 12$ Wochen)

- Upfront-Kombinationstherapie (zwischen dem Start beider Medikamente vergingen weniger als 12 Wochen).

Als primären Studienendpunkt definierten die Wissenschaftler den Tod aufgrund jeglicher Ursache 1, 3 bzw. 5 Jahre nach der Diagnose der pulmonalarteriellen Hypertonie. Ferner erfassten sie die Nebenwirkungen der medikamentösen Therapie. 


\section{Ergebnisse}

34 der 76 Patienten mit einer pulmonalarteriellen Hypertonie (45\%) erhielten eine Monotherapie: 22 (29\%) nahmen ERA und 12 (16\%) PDE5-Hemmer ein. 25 Personen (33\%) erhielten eine sequenzielle und 17 (22\%) eine Upfront-Kombinationstherapie. Die mittels sequenzieller Kombinationstherapie behandelten Patienten wiesen zu Behandlungsbeginn eine niedrigere FVC/DLCO (forcierte Vitalkapazität/CO-Diffusionskapazität)-Ratio auf und in den beiden KombinationstherapieGruppen stellten die Wissenschaftler initial einen schlechteren durchschnittlichen Pulmonalarteriendruck fest. Die multivariate Analyse ergab: Das verordnete Therapieregime sowie die initiale kardiale Funktion gemäß NYHA-Klassifikation stellten unabhängige Prädiktoren für die Mortalität dar. Im Vergleich zur Monotherapie erwies sich die sequenzielle Kombinationstherapie diesbezüglich als protektiv (Hazard Ratio 0,23; $95 \%$ KI 0,07-0,69). Die Upfront-Kombinationstherapie ging ebenfalls mit einem - jedoch statistisch nicht signifikanten - Überlebensvorteil einher. Die Überlebensraten bei pulmonalarterieller Hypertonie betrugen unter Monotherapie, sequenzieller bzw. UpfrontKombinationstherapie nach einem Jahr 78, 95,8 bzw. 94,1\%, nach 3 Jahren 40,7, 81,5 bzw. 51,8\% und nach 5 Jahren 31,6, 56,5 bzw. $34,5 \%$ ( $p=0,007)$. Im Hinblick auf Therapienebenwirkungen unterschieden sich die $3 \mathrm{~Pa}$ tientengruppen nicht wesentlich.

\section{FAZIT}

Die Forscher schlussfolgern: Patienten mit einer pulmonalarteriellen Hypertonie auf der Basis einer Systemsklerose profitieren hinsichtlich der Überlebensprognose von einer Kombinationstherapie aus ERA und PDE5-Hemmern, wobei die sequenzielle Kombinationstherapie gegenüber der Monotherapie offenbar die deutlichsten Vorteile hat. Angesichts der infausten Prognose des Krankheitsbilds müssen ihrer Ansicht nach nun Studien den Nutzen der beiden Kombinationstherapie-Regimes differenzierter beleuchten.

Dr. med. Judith Lorenz, Künzell 